\title{
FINITE ELEMENT DISCRETIZATION ERROR ANALYSIS OF A SURFACE TENSION FORCE IN TWO-PHASE INCOMPRESSIBLE FLOWS
}

\author{
SVEN GROß AND ARNOLD REUSKEN
}

\begin{abstract}
We consider a standard model for a stationary two-phase incompressible flow with surface tension. In the variational formulation of the model a linear functional which describes the surface tension force occurs. This functional depends on the location and the curvature of the (unknown) interface. In a finite element discretization method the functional has to be approximated. For an approximation method based on a Laplace-Beltrami representation of the curvature we derive sharp bounds for the approximation error. A new modified approximation method with a significantly smaller error is introduced.
\end{abstract}

AMS subject classifications. 65M60, 65N15, 65N30, 76D45, 76T99

1. Introduction. Let $\Omega \subset \mathbb{R}^{3}$ be a polyhedral domain that contains a flow of two different immiscible incompressible newtonian phases (fluid-fluid or fluid-gas). At the interface between the two phases there are surface tension forces that are significant and can not be neglected. An example is a (rising) liquid drop contained in a surrounding fluid. The standard model to describe such a flow problem consists of instationary Navier-Stokes equations with certain coupling conditions at the interface which describe the effect of surface tension. In this paper we analyze errors that are due to the discretization of the surface tension force that occurs in the continuous model. To simplify the presentation and the analysis we assume a stationary flow.

The domains which contain the phases are denoted by $\Omega_{1}$ and $\Omega_{2}$ with $\bar{\Omega}_{1} \cup \bar{\Omega}_{2}=\bar{\Omega}$ and $\partial \Omega_{1} \cap \partial \Omega=\emptyset$. The interface between the two phases $\left(\partial \Omega_{1} \cap \partial \Omega_{2}\right)$ is denoted by $\Gamma$. To model the forces at the interface we make the standard assumption that the surface tension balances the jump of the normal stress on the interface, i.e., we have an interface condition

$$
[\boldsymbol{\sigma} \mathbf{n}]_{\Gamma}=\tau \mathcal{K} \mathbf{n},
$$

with $\mathbf{n}=\mathbf{n}_{\Gamma}$ the unit normal at the interface (pointing from $\Omega_{1}$ in $\Omega_{2}$ ), $\tau$ the surface tension coefficient (material parameter), $\mathcal{K}$ the curvature of $\Gamma$ and $\boldsymbol{\sigma}$ the stress tensor, i. e.,

$$
\boldsymbol{\sigma}=-p \mathbf{I}+\mu \mathbf{D}(\mathbf{u}), \quad \mathbf{D}(\mathbf{u})=\nabla \mathbf{u}+(\nabla \mathbf{u})^{T},
$$

with $p=p(x, t)$ the pressure, $\mathbf{u}=\mathbf{u}(x, t)$ the velocity vector and $\mu$ the viscosity. We assume continuity of the velocity across the interface. In combination with the conservation laws of mass and momentum this yields the following standard model, cf., for example, [20, 19, 23, 22]:

$$
\begin{aligned}
& \left\{\begin{array}{ll}
-\operatorname{div}\left(\mu_{i} \mathbf{D}(\mathbf{u})\right)+\rho_{i}(\mathbf{u} \cdot \nabla) \mathbf{u}-\nabla p=\rho_{i} \mathbf{g} & \text { in } \Omega_{i} \\
\operatorname{div} \mathbf{u}=0 & \text { in } \Omega_{i}
\end{array} \text { for } i=1,2\right. \\
& {[\boldsymbol{\sigma} \mathbf{n}]_{\Gamma}=\tau \mathcal{K} \mathbf{n}, \quad[\mathbf{u}]_{\Gamma}=0 .}
\end{aligned}
$$

*Institut für Geometrie und Praktische Mathematik, RWTH Aachen, D-52056 Aachen, Germany; email: reusken@igpm.rwth-aachen.de. This work was supported by the German Research Foundation through SFB 540 
The vector $\mathbf{g}$ is a known external force (gravity). In addition we need boundary conditions for $\mathbf{u}$ at $\partial \Omega$. For simplicity we take homogeneous Dirichlet boundary conditions. The two Navier-Stokes equations in (1.1) and the coupling conditons at the interface in (1.2) can be reformulated in one Navier-Stokes equation in the whole domain in which the effect of the surface tension is expressed in terms of a localized force at the interface, $c f$. the so-called continuum surface force (CSF) model [3, 4]. We consider this alternative formulation in a standard weak form (as in [10, 24, 25, 26, 27]) in the spaces

$$
\mathbf{V}:=H_{0}^{1}(\Omega)^{3}, \quad Q:=L_{0}^{2}(\Omega)=\left\{q \in L^{2}(\Omega) \mid \int_{\Omega} q d x=0\right\} .
$$

For the $L^{2}$ scalar product we use the notation $(f, g):=\int_{\Omega} f g d x$ (and similarly for vector functions). The standard norm in $\mathbf{V}$ is denoted by $\|\cdot\|_{1}$. The weak formulation is as follows: determine $(\mathbf{u}, p) \in \mathbf{V} \times Q$ such that

$$
\begin{aligned}
\int_{\Omega} \mu \mathbf{D}(\mathbf{u}): \mathbf{D}(\mathbf{v}) d x+(\rho \mathbf{u} \cdot \nabla \mathbf{u}, \mathbf{v})+(\operatorname{div} \mathbf{v}, p) & =(\rho \mathbf{g}, \mathbf{v})+f_{\Gamma}(\mathbf{v}) \quad \forall \mathbf{v} \in \mathbf{V} \\
(\operatorname{div} \mathbf{u}, q) & =0 \quad \forall q \in Q,
\end{aligned}
$$

with

$$
f_{\Gamma}(\mathbf{v})=\tau \int_{\Gamma} \mathcal{K}_{\mathbf{n}_{\Gamma}} \cdot \mathbf{v} d s
$$

and $\mathbf{D}(\mathbf{u}): \mathbf{D}(\mathbf{v})=\operatorname{tr}((\mathbf{D}(\mathbf{u}) \mathbf{D}(\mathbf{v}))$. The functions $\mu$ and $\rho$ are strictly positive and piecewise constant in $\Omega_{i}, i=1,2$. For $\Gamma$ sufficiently smooth we have $\sup _{x \in \Gamma}|\mathcal{K}(x)| \leq$ $c<\infty$ and thus

$$
\left|f_{\Gamma}(\mathbf{v})\right| \leq c \tau \int_{\Gamma}\left|\mathbf{n}_{\Gamma} \cdot \mathbf{v}\right| d s \leq c\|\mathbf{v}\|_{L^{2}(\Gamma)} \leq c\|\mathbf{v}\|_{1} \quad \text { for all } \mathbf{v} \in \mathbf{V} .
$$

Here and in the remainder we use the notation $c$ for a generic constant. ¿From (1.5) we see that $f_{\Gamma} \in \mathbf{V}^{\prime}$ and thus under the usual assumptions (cf. [11]) the stationary Navier-Stokes equations (1.3) have a unique solution. We emphasize that the location of the interface is in general unknown and has to be determined (approximated) before the Navier-Stokes equations (1.3) can be solved. In this paper we assume that the unknown interface is captured using a level set technique. For a discussion of level set methods in incompressible two-phase flow problems we refer to the literature $[4,12,18,21]$. We assume that the interface $\Gamma$ is characterized as the zero level of the level set function $d$, which locally (close to the interface) is a signed distance function.

We now turn to the discretization of (1.3). We assume that $\mathcal{S}$ is a triangulation of $\Omega$ consisting of tetrahedra. With this triangulation we associate a mesh size parameter $H$. Let $\mathbf{V}_{H} \subset \mathbf{V}, Q_{H} \subset Q$ be standard polynomial finite element spaces corresponding to the triangulation $\mathcal{S}$, for example, the Hood-Taylor $P_{2}-P_{1}$ pair. In practice, the triangulation $\mathcal{S}$ is locally refined close to the interface $\Gamma$ but not aligned with this interface, cf. Figures 2.1, 6.1. The Galerkin discretization is as follows: determine $\left(\mathbf{u}_{H}, p_{H}\right) \in \mathbf{V}_{H} \times Q_{H}$ such that

$$
\begin{aligned}
& \int_{\Omega} \mu \mathbf{D}\left(\mathbf{u}_{H}\right): \mathbf{D}\left(\mathbf{v}_{H}\right) d x+\left(\rho \mathbf{u}_{H} \cdot \nabla \mathbf{u}_{H}, \mathbf{v}_{H}\right)+\left(\operatorname{div} \mathbf{v}_{H}, p_{H}\right) \\
& =\left(\rho \mathbf{g}, \mathbf{v}_{H}\right)+f_{\Gamma}\left(\mathbf{v}_{H}\right) \text { for all } \mathbf{v}_{H} \in \mathbf{V}_{H} \\
& \left(\operatorname{div} \mathbf{u}_{H}, q_{H}\right)=0 \text { for all } q_{H} \in Q_{H} .
\end{aligned}
$$


For this discrete problem, many important theoretical issues are still unsolved. For example, regarding iterative solvers there is the issue of robustness w.r.t. large jumps in the density and viscosity coefficients (results for Stokes equations are given in $[17,16,15])$. A second example is the effect of errors in the approximation of $f_{\Gamma}\left(\mathbf{v}_{H}\right)$ on the accuracy of the flow variables. In this paper we treat the latter topic.

As mentioned above, the interface $\Gamma$ has to be approximated. Furthermore, to evaluate the integral in (1.4) the curvature of $\Gamma$ has to be approximated and a quadrature rule may be needed. Thus the term $f_{\Gamma}\left(\mathbf{v}_{H}\right)$ on the right handside in (1.6) will be replaced by an approximation $\tilde{f}\left(\mathbf{v}_{H}\right)$. For the effect of the surface tension force approximation error on the accuracy of the velocity and pressure variables the quantity

$$
\sup _{\mathbf{v} \in \mathbf{V}_{H}} \frac{f_{\Gamma}\left(\mathbf{v}_{H}\right)-\tilde{f}\left(\mathbf{v}_{H}\right)}{\left\|\mathbf{v}_{H}\right\|_{1}}
$$

is crucial (Strang lemma). The two main ingredients in the approximation method that we use are the following. Firstly, a Laplace-Beltrami characterization of the curvature is used. This technique has been applied in mean curvature flows (cf. [5]), and in flows with a free capillary surface (cf. [1, 2]). Application of this technique in two-phase incompressible flows can be found in $[10,9,12,14]$. Secondly, the unknown interface $\Gamma$ (zero level of $d$ ) is approximated as the zero level $\Gamma_{h}$ of a finite element approximation $d_{h}$ of $d$. The approximate interface $\Gamma_{h}$ consists of triangular faces. The parameter $h$ is the maximal diameter of these faces and is not necessarily of the same order of magnitude as $H$. For this approximation technique we derive a sharp bound for the quantity in (1.7). The main result of this paper is the $\mathcal{O}(\sqrt{h})$ bound given in Corollary 4.8. We do not know of any literature in which for this technique or for any other technique for approximating $f_{\Gamma}\left(\mathbf{v}_{H}\right)$ rigorous bounds for the quantity in (1.7) are derived. A numerical experiment (given in section 6) indicates that the $\mathcal{O}(\sqrt{h})$ is sharp. Our analysis reveals how the approximation method can be improved. A modified new approach, resulting in an $\mathcal{O}(h)$ bound, is presented in section 5 .

2. Approximation of the surface tension force $f_{\Gamma}\left(\mathbf{v}_{H}\right)$. In this section we explain how the localized surface tension force term, $f_{\Gamma}\left(\mathbf{v}_{H}\right)$ in (1.6), is approximated. For this we first need some notions from differential geometry.

Let $U$ be an open subset in $\mathbb{R}^{3}$ and $\Gamma$ a connected $C^{2}$ compact hypersurface contained in $U$. For a sufficiently smooth function $g: U \rightarrow \mathbb{R}$ the tangential derivative (along $\Gamma$ ) is defined by projecting the derivative on the tangent space of $\Gamma$, i. e.

$$
\nabla_{\Gamma} g=\nabla g-\nabla g \cdot \mathbf{n}_{\Gamma} \mathbf{n}_{\Gamma}
$$

The Laplace-Beltrami operator of $g$ on $\Gamma$ is defined by

$$
\Delta_{\Gamma} g:=\nabla_{\Gamma} \cdot \nabla_{\Gamma} g
$$

It can be shown that $\nabla_{\Gamma} g$ and $\Delta_{\Gamma} g$ depend only on values of $g$ on $\Gamma$. For vector valued functions $f, g: \Gamma \rightarrow \mathbb{R}^{3}$ we define

$$
\Delta_{\Gamma} f:=\left(\Delta_{\Gamma} f_{1}, \Delta_{\Gamma} f_{2}, \Delta_{\Gamma} f_{3}\right)^{T}, \quad \nabla_{\Gamma} f \cdot \nabla_{\Gamma} g:=\sum_{i=1}^{3} \nabla_{\Gamma} f_{i} \cdot \nabla_{\Gamma} g_{i} .
$$

We recall the following basic result from differential geometry. 
Theorem 2.1. Let $\operatorname{id}_{\Gamma}: \Gamma \rightarrow \mathbb{R}^{3}$ be the identity on $\Gamma$ and $\mathcal{K}=\kappa_{1}+\kappa_{2}$ the sum of the principal curvatures. For all sufficiently smooth vector functions $\mathbf{v}$ on $\Gamma$ the following holds:

$$
\int_{\Gamma} \mathcal{K} \mathbf{n}_{\Gamma} \cdot \mathbf{v} d s=-\int_{\Gamma}\left(\Delta_{\Gamma} \mathrm{id}_{\Gamma}\right) \cdot \mathbf{v} d s=\int_{\Gamma} \nabla_{\Gamma} \operatorname{id}_{\Gamma} \cdot \nabla_{\Gamma} \mathbf{v} d s
$$

In a finite element setting (which is based on a weak formulation) it is natural to use the expression on the right handside in $(2.2)$ as a starting point for the discretization. This idea is used in, for example, $[8,2,10,12]$. In this discretization we use an approximation $\Gamma_{h}$ of $\Gamma$. Given this approximation the localized force term $f_{\Gamma}\left(\mathbf{v}_{H}\right)$ is approximated by

$$
\tilde{f}\left(\mathbf{v}_{H}\right)=f_{\Gamma_{h}}\left(\mathbf{v}_{H}\right):=\tau \int_{\Gamma_{h}} \nabla_{\Gamma_{h}} \operatorname{id}_{\Gamma_{h}} \cdot \nabla_{\Gamma_{h}} \mathbf{v}_{H} d s .
$$

We now introduce some additional notation and formulate assumptions on $\Gamma_{h}$. In Remark 3 we explain how in practice a discrete interface $\Gamma_{h}$ can be computed which satisfies these assumptions.

We assume that $\Gamma$ is the zero level set of a signed distance function $d: U \rightarrow \mathbb{R}$, i.e., $|d(x)|=\operatorname{dist}(x, \Gamma)$ for all $x \in U$. We assume $d<0$ on the interior of $\Gamma$ (that is, in $\left.\Omega_{1}\right)$ and $d>0$ on the exterior. Note that $\mathbf{n}_{\Gamma}=\nabla d$ on $\Gamma$. We define $\mathbf{n}(x):=\nabla d(x)$ for all $x \in U$. Thus $\mathbf{n}=\mathbf{n}_{\Gamma}$ on $\Gamma$ and $\|\mathbf{n}(x)\|=1$ for all $x \in U$. Here and in the remainder $\|\cdot\|$ denotes the Euclidean norm. The Hessian of $d$ is denoted by $\mathbf{H}$ :

$$
\mathbf{H}(x)=D^{2} d(x) \in \mathbb{R}^{3 \times 3} \quad \text { for all } x \in U .
$$

The eigenvalues of $\mathbf{H}(x)$ are denoted by $\kappa_{1}(x), \kappa_{2}(x)$ and 0 . For $x \in \Gamma$ the eigenvalues $\kappa_{i}(x), i=1,2$, are the principal curvatures.

We will need the orthogonal projection

$$
\mathbf{P}(x)=\mathbf{I}-\mathbf{n}(x) \mathbf{n}(x)^{T} \quad \text { for } x \in U .
$$

Note that the tangential derivative can be written as $\nabla_{\Gamma} g=\mathbf{P} \nabla g$. Let $\left\{\Gamma_{h}\right\}_{h>0}$ be a family of polyhedrons. Each $\Gamma_{h}$ is contained in $U$ and consists of a set $\mathcal{F}_{h}$ of triangular faces: $\Gamma_{h}=\cup_{T \in \mathcal{F}_{h}} T$. For $T_{1}, T_{2} \in \mathcal{F}_{h}$ with $T_{1} \neq T_{2}$ we assume that $T_{1} \cap T_{2}$ is either empty or a common edge or a common vertex. The parameter $h$ denotes the maximal diameter of the triangles in $\mathcal{F}_{h}: h=\max _{T \in \mathcal{F}_{h}} \operatorname{diam}(T)$. By $\mathbf{n}_{h}$ we denote the outward pointing unit normal on $\Gamma_{h}$. This normal is piecewise constant with possible discontinuities at the edges of the triangles in $\mathcal{F}_{h}$.

The approximation $\Gamma_{h}$ is assumed to be close to $\Gamma$ in the following sense:

$$
\begin{aligned}
& |d(x)| \leq c h^{2} \quad \text { for all } x \in \Gamma_{h}, \\
& \operatorname{essinf}_{x \in \Gamma_{h}} \mathbf{n}(x)^{T} \mathbf{n}_{h}(x) \geq c>0, \\
& \operatorname{ess} \sup _{x \in \Gamma_{h}}\left\|\mathbf{P}(x) \mathbf{n}_{h}(x)\right\| \leq \text { ch. }
\end{aligned}
$$

Here $c$ denotes a generic constant independent of $h$.

REMARK 1. The conditions (2.5), (2.6) are satisfied if

$$
\operatorname{ess}_{\sup _{x \in \Gamma_{h}}}\left\|\mathbf{n}(x)-\mathbf{n}_{h}(x)\right\| \leq \min \left\{c_{0}, c h\right\}, \quad \text { with } c_{0}<\sqrt{2},
$$


holds. This easily follows from

$$
\left\|\mathbf{n}(x)-\mathbf{n}_{h}(x)\right\|^{2}=2\left(1-\mathbf{n}(x)^{T} \mathbf{n}_{h}(x)\right)
$$

and

$$
\left\|\mathbf{P}(x) \mathbf{n}_{h}(x)\right\|=\left\|\mathbf{P}(x)\left(\mathbf{n}(x)-\mathbf{n}_{h}(x)\right)\right\| \leq\left\|\mathbf{n}(x)-\mathbf{n}_{h}(x)\right\| .
$$

Under these assumptions on $\Gamma_{h}$ we will derive, in section 4 , a bound for the approximation error

$$
\sup _{\mathbf{v}_{H} \in \mathbf{V}_{H}} \frac{f_{\Gamma}\left(\mathbf{v}_{H}\right)-f_{\Gamma_{h}}\left(\mathbf{v}_{H}\right)}{\left\|\mathbf{v}_{H}\right\|_{1}}, \quad \text { with } f_{\Gamma_{h}}\left(\mathbf{v}_{H}\right) \quad \text { as in (2.3). }
$$

REMARK 2. From Theorem 2.1, the fact that $f_{\Gamma}(\mathbf{v})=\tau \int_{\Gamma} \mathcal{K} \mathbf{v} \cdot \mathbf{n} d s$ is a bounded linear functional on $\mathbf{V}$ and a density argument it follows that the linear functional

$$
f_{\Gamma}: \mathbf{v} \rightarrow \tau \int_{\Gamma} \nabla_{\Gamma} \mathrm{id}_{\Gamma} \cdot \nabla_{\Gamma} \mathbf{v} d s, \quad \mathbf{v} \in\left(C_{0}^{\infty}(\Omega)\right)^{3},
$$

has a unique bounded extension to $\mathbf{V}$. Therefore, for $f_{\Gamma}: \mathbf{V} \rightarrow \mathbb{R}$ we can use both the representation in (1.4) and the one in (2.9) (these are the same on a dense subset). This, however, is not the case for $f_{\Gamma_{h}}$. Because $\Gamma_{h}$ is not sufficiently smooth, a partial integration result as in Theorem 2.1 does not hold. The linear functional

$$
\mathbf{v} \rightarrow \tau \int_{\Gamma_{h}} \nabla_{\Gamma_{h}} \operatorname{id}_{\Gamma_{h}} \cdot \nabla_{\Gamma_{h}} \mathbf{v} d s
$$

is not necessarily bounded on $\mathbf{V}$. For this reason the restriction to $\mathbf{v}_{H}$ from the finite element space $\mathbf{V}_{H}$ in (2.8) is essential.

REMARK 3. We briefly explain the approach that is used in [12] (cf. also [7]) for computing $\Gamma_{h}$. Let $\mathcal{S}$ be the (locally refined) triangulation of $\Omega$, consisting of tetrahedra, that is used for the discretization of the flow variables with finite elements, cf. (1.6) (in our approach we use the Hood-Taylor $P_{2}-P_{1}$ pair). The level set equation for $d$ is discretized with continuous piecewise quadratic finite elements on a triangulation $\mathcal{T}$. This triangulation is either equal to $\mathcal{S}$ or obtained from one or a few regular refinements of $\mathcal{S}$ (subdivision of each tetrahedron in 8 child tetrahedra). The piecewise quadratic finite element approximation of $d$ on $\mathcal{T}$ is denoted by $d_{h}$. We now introduce one further regular refinement of $\mathcal{T}$, resulting in $\mathcal{T}^{\prime}$. Let $I\left(d_{h}\right)$ be the continuous piecewise linear function on $\mathcal{T}^{\prime}$ which interpolates $d_{h}$ at all vertices of all tetrahedra in $\mathcal{T}^{\prime}$. The approximation of the interface $\Gamma$ is defined by

$$
\Gamma_{h}:=\left\{x \in \Omega \mid I\left(d_{h}\right)(x)=0\right\}
$$

which consists of piecewise planar segments. The mesh size parameter $h$ is the maximal diameter of these segments. This (maximal) diameter is approximately the (maximal) diameter of the tetrahedra in $\mathcal{T}^{\prime}$ that contain the discrete interface, i.e., $h$ is approximately the maximal diameter of the tetrahedra in $\mathcal{T}^{\prime}$ that are close to the interface. In Figure 2.1 we illustrate this construction for the two-dimensional case.

Each of the planar segments of $\Gamma_{h}$ is either a triangle or a quadrilateral. The quadrilaterals can (formally) be divided into two triangles. Thus $\Gamma_{h}$ consists of a set 


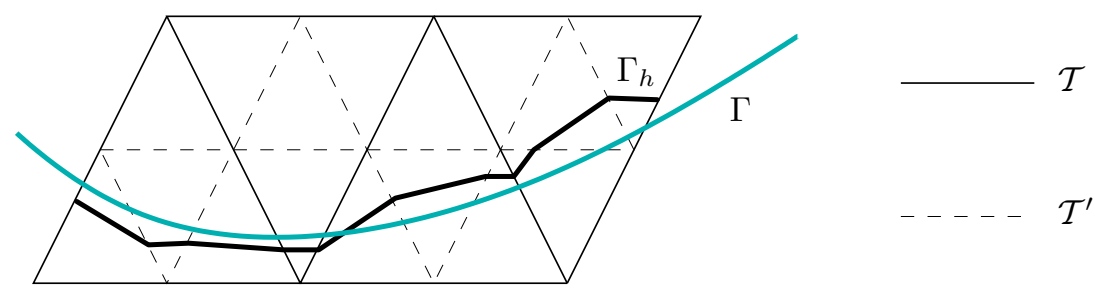

FIG. 2.1. Construction of approximate interface for $2 D$ case.

$\mathcal{F}_{h}$ of triangular faces. Related to the assumptions (2.4)-(2.6) we note the following. If we assume $\left|I\left(d_{h}\right)(x)-d(x)\right| \leq c h^{2}$ for all $x$ in a neighbourhood of $\Gamma$, which is reasonable for a smooth $d$ and piecewise quadratic $d_{h}$, then for $x \in \Gamma_{h}$ we have $|d(x)|=\left|d(x)-I\left(d_{h}\right)(x)\right| \leq c h^{2}$ and thus (2.4) is satisfied. Instead of (2.5), (2.6) we consider the sufficient condition (2.7). We assume $\left\|\nabla d(x)-\nabla I\left(d_{h}\right)(x)\right\| \leq c h$ for all $x$ in a neighbourhood of $\Gamma$ ( $x$ not on an edge), which again is reasonable for a smooth $d$ and piecewise quadratic $d_{h}$. Due to $\|\nabla d\|=1$ we then also have $\left\|\nabla I\left(d_{h}\right)(x)\right\|=1+\mathcal{O}(h)$, in a neighbourhood of $\Gamma$. For $x \in \Gamma_{h}$ (not on an edge) we obtain

$$
\begin{aligned}
\left\|\mathbf{n}_{h}(x)-\mathbf{n}(x)\right\| & =\left\|\frac{\nabla I\left(d_{h}\right)(x)}{\left\|\nabla I\left(d_{h}\right)(x)\right\|}-\nabla d(x)\right\| \\
& \leq\left|\frac{1}{\left\|\nabla I\left(d_{h}\right)(x)\right\|}-1\right| \cdot\left\|\nabla I\left(d_{h}\right)(x)\right\|+\left\|\nabla I\left(d_{h}\right)(x)-\nabla d(x)\right\| \leq c h,
\end{aligned}
$$

and thus (2.7) is satisfied (for $h$ sufficiently small).

3. Preliminaries. In this section we collect some results that will be used in the analysis in section 4. The techniques that we use come from the paper [6]. For proofs of certain results we will refer to that paper.

We introduce a locally (in a neighborhood of $\Gamma$ ) orthogonal coordinate system by using the projection $\mathbf{p}: U \rightarrow \Gamma$ :

$$
\mathbf{p}(x)=x-d(x) \mathbf{n}(x) \text { for all } x \in U .
$$

We assume that the decomposition $x=\mathbf{p}(x)+d(x) \mathbf{n}(x)$ is unique for all $x \in U$. Note that

$$
\mathbf{n}(x)=\mathbf{n}(\mathbf{p}(x)) \text { for all } x \in U \text {. }
$$

We use an extension operator defined as follows. For a (scalar) function $v$ defined on $\Gamma$ we define

$$
v_{\Gamma}^{e}(x):=v(x-d(x) \mathbf{n}(x))=v(\mathbf{p}(x)) \text { for all } x \in U,
$$

i.e., $v$ is extended along normals on $\Gamma$. We will also need extensions of functions defined on $\Gamma_{h}$ to $U$. This is done again by extending along normals $\mathbf{n}(x)$. For $v$ defined on $\Gamma_{h}$ we define, for $x \in \Gamma_{h}$,

$$
v_{\Gamma_{h}}^{e}(x+\alpha \mathbf{n}(x)):=v(x) \text { for all } \alpha \in \mathbb{R} \quad \text { with } x+\alpha \mathbf{n}(x) \in U .
$$

The projection $\mathbf{p}$ and the extensions $v_{\Gamma}^{e}, v_{\Gamma_{h}}^{e}$ are illustrated in Figure 3.1. 


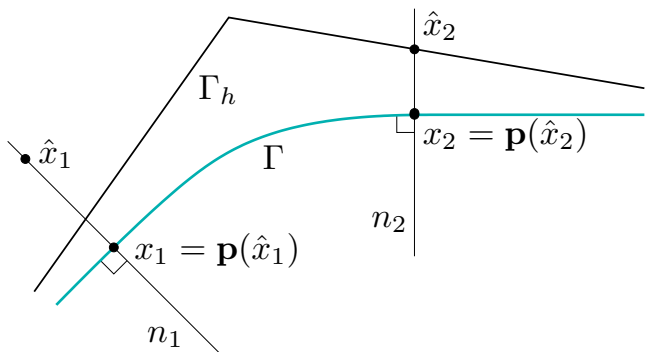

Fig. 3.1. Example for projection $\mathbf{p}$ and construction of extension operators. $n_{1}$ and $n_{2}$ are straight lines perpendicular to $\Gamma$. For $v$ defined on $\Gamma$ we have $v_{\Gamma}^{e} \equiv v\left(x_{1}\right)$ on $n_{1}$. For $v_{h}$ defined on $\Gamma_{h}$ we have $v_{\Gamma_{h}}^{e} \equiv v_{h}\left(\hat{x}_{2}\right)$ on $n_{2}$.

We define a discrete analogon of the orthogonal projection $\mathbf{P}$ :

$$
\mathbf{P}_{h}(x):=\mathbf{I}-\mathbf{n}_{h}(x) \mathbf{n}_{h}(x)^{T} \text { for } x \in \Gamma_{h}, x \text { not on an edge. }
$$

The tangential derivative along $\Gamma_{h}$ can be written as $\nabla_{\Gamma_{h}} g=\mathbf{P}_{h} \nabla g$. In the analysis a further technical assumption is used, namely that the neighbourhood $U$ of $\Gamma$ is sufficiently small in the following sense. We assume that $U$ is a strip of width $\delta>0$ with

$$
\delta^{-1}>\max _{i=1,2}\left\|\kappa_{i}(x)\right\|_{L^{\infty}(\Gamma)}
$$

Assumption 1. In the remainder of the paper we assume that (2.4), (2.5), (2.6)and (3.2) hold.

We present two lemmas from [6]. Proofs are elementary and can be found in [6].

Lemma 3.1. For the projection operator $\mathbf{P}$ and the Hessian $\mathbf{H}$ the relation

$$
\mathbf{P}(x) \mathbf{H}(x)=\mathbf{H}(x) \mathbf{P}(x)=\mathbf{H}(x) \quad \text { for all } \quad x \in U
$$

holds. For $v$ defined on $\Gamma$ and sufficiently smooth the following holds:

$$
\nabla_{\Gamma_{h}} v_{\Gamma}^{e}(x)=\mathbf{P}_{h}(x)(\mathbf{I}-d(x) \mathbf{H}(x)) \mathbf{P}(x) \nabla_{\Gamma} v(\mathbf{p}(x)) \quad \text { a.e. on } \Gamma_{h} .
$$

Proof. Given in section 2.3 in [6]. $\mathrm{\square}$

In (3.3) (and also below) we have results "a.e. on $\Gamma_{h}$ " because quantities (derivatives, $\mathbf{P}_{h}$, etc.) are not well-defined on the edges of the triangulation $\Gamma_{h}$.

Lemma 3.2. For $x \in \Gamma_{h}$ (not on an edge) define

$$
\begin{aligned}
\mu(x) & =\left[\Pi_{i=1}^{2}\left(1-d(x) \kappa_{i}(x)\right)\right] \mathbf{n}(x)^{T} \mathbf{n}_{h}(x), \\
\mathbf{A}(x) & =\frac{1}{\mu(x)} \mathbf{P}(x)[\mathbf{I}-d(x) \mathbf{H}(x)] \mathbf{P}_{h}(x)[\mathbf{I}-d(x) \mathbf{H}(x)] \mathbf{P}(x) .
\end{aligned}
$$

Let $\mathbf{A}_{\Gamma_{h}}^{e}$ be the extension of $\mathbf{A}$ as in (3.1). The following identity holds for functions $v$ and $\psi$ that are defined on $\Gamma_{h}$ and sufficiently smooth:

$$
\int_{\Gamma_{h}} \nabla_{\Gamma_{h}} v \cdot \nabla_{\Gamma_{h}} \psi d s=\int_{\Gamma} \mathbf{A}_{\Gamma_{h}}^{e} \nabla_{\Gamma} v_{\Gamma_{h}}^{e} \cdot \nabla_{\Gamma} \psi_{\Gamma_{h}}^{e} d s
$$


Proof. Given in section 2.3 in [6]. $\square$

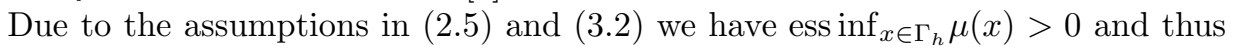
$\mathbf{A}(x)$ is well-defined.

We now derive two further results that are needed in the analysis in section 4 .

LEMma 3.3. There exists a constant $c$ independent of $h$ such that

$$
\left\|\nabla_{\Gamma} v_{\Gamma_{h}}^{e}\right\|_{L^{2}(\Gamma)} \leq c\left\|\nabla_{\Gamma_{h}} v\right\|_{L^{2}\left(\Gamma_{h}\right)} \quad \text { for all } v \in H^{1}\left(\Gamma_{h}\right) \cap C\left(\Gamma_{h}\right) .
$$

Proof. Due to Lemma 3.2 we have

$$
\left\|\nabla_{\Gamma_{h}} v\right\|_{L^{2}\left(\Gamma_{h}\right)}^{2}=\int_{\Gamma} \mathbf{A}_{\Gamma_{h}}^{e}(y) \nabla_{\Gamma} v_{\Gamma_{h}}^{e}(y) \cdot \nabla_{\Gamma} v_{\Gamma_{h}}^{e}(y) d s(y)
$$

with $d s(y)$ the surface measure on $\Gamma$. Take $x \in \Gamma_{h}$ with $\mathbf{p}(x)=y$. If $x$ does not lie on an edge, we have $\mathbf{A}_{\Gamma_{h}}^{e}(y)=\mathbf{A}(x)$ with $\mathbf{A}(x)$ as in (3.5). We drop the symbol $x$ in the notation and write $\mathbf{A}(x)=\mathbf{A}=\frac{1}{\mu} \mathbf{P}(\mathbf{I}-d \mathbf{H}) \mathbf{P}_{h}(\mathbf{I}-d \mathbf{H}) \mathbf{P}$. Decompose $\mathbf{n}_{h}$ as $\mathbf{n}_{h}=\alpha \mathbf{n}+\beta \mathbf{n}^{\perp}$ with $\left\|\mathbf{n}^{\perp}\right\|=1$ and $\mathbf{n}^{T} \mathbf{n}^{\perp}=0$. From (2.5) is follows that $\alpha \geq c>0$ and thus $\beta^{2} \leq 1-c^{2}<1$. Take $z \in \operatorname{range}(\mathbf{P})$ with $\|z\|=1$. We then have $\left\|\mathbf{P}_{h} z\right\| \geq\|z\|-\left|z^{T} \mathbf{n}_{h}\right|=\|z\|-|\beta|\left|z^{T} \mathbf{n}^{\perp}\right| \geq(1-|\beta|)\|z\|$. Hence, there is a constant $c>0$ such that

$$
\left\|\mathbf{P}_{h} \mathbf{P} w\right\| \geq c\|\mathbf{P} w\| \quad \text { for all } w \in \mathbb{R}^{3} .
$$

Using (3.2) it follows that there is a constant $c>0$ such that $\|(\mathbf{I}-d \mathbf{H}) w\| \geq c\|w\|$ for all $w \in \mathbb{R}^{3}$. Note that $\mu=\mu(x) \geq c>0$ holds. From these results we obtain, using $\mathbf{P H}=\mathbf{H P}$ (Lemma 3.1),

$$
\begin{aligned}
w^{T} \mathbf{A} w & =\frac{1}{\mu} w^{T} \mathbf{P}(\mathbf{I}-d \mathbf{H}) \mathbf{P}_{h}(\mathbf{I}-d \mathbf{H}) \mathbf{P} w \\
& =\frac{1}{\mu}\left\|\mathbf{P}_{h} \mathbf{P}(\mathbf{I}-d \mathbf{H}) w\right\|^{2} \geq c\|\mathbf{P} w\|^{2} \quad \text { for all } w \in \mathbb{R}^{3},
\end{aligned}
$$

with a constant $c>0$. This yields, using $\mathbf{n}(x)=\mathbf{n}(\mathbf{p}(x))=\mathbf{n}(y)$,

$$
\mathbf{A}_{\Gamma_{h}}^{e}(y) w \cdot w=w^{T} \mathbf{A}(x) w \geq c\|\mathbf{P}(x) w\|^{2}=\|\mathbf{P}(y) w\|^{2},
$$

with $c>0$. For $w=\nabla_{\Gamma} v_{\Gamma_{h}}^{e}(y)$ we have $\mathbf{P}(y) w=\mathbf{P}(y) \nabla_{\Gamma} v_{\Gamma_{h}}^{e}(y)=\nabla_{\Gamma} v_{\Gamma_{h}}^{e}(y)$ and thus we get

$$
\begin{aligned}
\left\|\nabla_{\Gamma_{h}} v\right\|_{L^{2}\left(\Gamma_{h}\right)}^{2} & =\int_{\Gamma} \mathbf{A}_{\Gamma_{h}}^{e}(y) \nabla_{\Gamma} v_{\Gamma_{h}}^{e}(y) \cdot \nabla_{\Gamma} v_{\Gamma_{h}}^{e}(y) d s(y) \\
& \geq c \int_{\Gamma} \nabla_{\Gamma} v_{\Gamma_{h}}^{e}(y) \cdot \nabla_{\Gamma} v_{\Gamma_{h}}^{e}(y) d s(y)=c\left\|\nabla_{\Gamma} v_{\Gamma_{h}}^{e}\right\|_{L^{2}(\Gamma)}^{2},
\end{aligned}
$$

with a constant $c>0$.

Lemma 3.4. The following holds:

$$
\operatorname{ess} \sup _{y \in \Gamma}\left\|\left(\mathbf{A}_{\Gamma_{h}}^{e}(y)-\mathbf{I}\right) \mathbf{P}(y)\right\| \leq c h^{2} .
$$


Proof. Take $y \in \Gamma$ and a corresponding $x \in \Gamma_{h}$ such that $\mathbf{p}(x)=y$. Assume that $x$ does not lie on an edge of the triangulation $\Gamma_{h}$, which is true for almost all $y \in \Gamma$. Then we have

$$
\left(\mathbf{A}_{\Gamma_{h}}^{e}(y)-\mathbf{I}\right) \mathbf{P}(y)=(\mathbf{A}(x)-\mathbf{I}) \mathbf{P}(x) .
$$

We drop the symbol $x$ in the notation and write $\mathbf{A}(x)=\mathbf{A}=\frac{1}{\mu} \mathbf{P}(\mathbf{I}-d \mathbf{H}) \mathbf{P}_{h}(\mathbf{I}-d \mathbf{H}) \mathbf{P}$. Note that $|\mu|=\mu(x) \geq c>0$ holds. Decompose $\mathbf{n}_{h}$ as $\mathbf{n}_{h}=\alpha \mathbf{n}+\beta \mathbf{n}^{\perp}$ with $\left\|\mathbf{n}^{\perp}\right\|=1$ and $\mathbf{n}^{T} \mathbf{n}^{\perp}=0$. Due to (2.5) we have $\alpha=\mathbf{n}^{T} \mathbf{n}_{h} \geq c>0$. From (2.6) we get $\left\|\mathbf{P n}_{h}\right\|=|\beta| \leq$ ch. Hence,

$$
\left|\mathbf{n}^{T} \mathbf{n}_{h}-1\right|=1-\alpha=\frac{1-\alpha^{2}}{1+\alpha} \leq 1-\alpha^{2}=\beta^{2} \leq c h^{2} .
$$

Using this and $|d(x)| \leq c h^{2},\left|\kappa_{i}(x)\right| \leq c$, we obtain $|\mu-1| \leq c h^{2}$. Thus

$$
\left|\frac{1}{\mu}-1\right|=\frac{|\mu-1|}{\mu} \leq c h^{2}
$$

holds. We have

$$
\begin{aligned}
& (\mathbf{A}-\mathbf{I}) \mathbf{P}=\frac{1}{\mu} \mathbf{P}(\mathbf{I}-d \mathbf{H}) \mathbf{P}_{h}(\mathbf{I}-d \mathbf{H}) \mathbf{P}-\mathbf{P} \\
& =\left[\left(\frac{1}{\mu}-1\right) \mathbf{P}(\mathbf{I}-d \mathbf{H}) \mathbf{P}_{h}(\mathbf{I}-d \mathbf{H}) \mathbf{P}\right]+\left[\mathbf{P}(\mathbf{I}-d \mathbf{H}) \mathbf{P}_{h}(\mathbf{I}-d \mathbf{H}) \mathbf{P}-\mathbf{P}\right]
\end{aligned}
$$

and consider the two terms on the right handside separately. For the first term we get, using (3.8),

$$
\left\|\left(\frac{1}{\mu}-1\right) \mathbf{P}(\mathbf{I}-d \mathbf{H}) \mathbf{P}_{h}(\mathbf{I}-d \mathbf{H}) \mathbf{P}\right\| \leq\left|\frac{1}{\mu}-1\right|\left(1+c h^{2}\right)\left(1+c h^{2}\right) \leq c h^{2} .
$$

For the second term we obtain, using (2.6),

$$
\begin{aligned}
& \left\|\mathbf{P}(\mathbf{I}-d \mathbf{H}) \mathbf{P}_{h}(\mathbf{I}-d \mathbf{H}) \mathbf{P}-\mathbf{P}\right\| \leq\left\|\mathbf{P} \mathbf{P}_{h} \mathbf{P}-\mathbf{P}\right\|+c h^{2} \\
& =\left\|\mathbf{P n}_{h} \mathbf{n}_{h}^{T} \mathbf{P}\right\|+c h^{2}=\left\|\mathbf{P n}_{h}\right\|^{2}+c h^{2} \leq c h^{2} .
\end{aligned}
$$

Combination of these bounds completes the proof.

4. Approximation error analysis. We are interested in the difference between the terms

$$
\tau \int_{\Gamma} \nabla_{\Gamma} \operatorname{id}_{\Gamma} \cdot \nabla_{\Gamma} \mathbf{v}_{H} d s \quad \text { and } \tau \int_{\Gamma_{h}} \nabla_{\Gamma_{h}} \operatorname{id}_{\Gamma_{h}} \cdot \nabla_{\Gamma_{h}} \mathbf{v}_{H} d s \text { for } \mathbf{v}_{H} \in \mathbf{V}_{H} .
$$

Since $\nabla_{\Gamma} \mathrm{id}_{\Gamma} \cdot \nabla_{\Gamma} \mathbf{v}_{H}=\sum_{i=1}^{3} \nabla_{\Gamma}\left(\mathrm{id}_{\Gamma}\right)_{i} \cdot \nabla_{\Gamma}\left(\mathbf{v}_{H}\right)_{i}$ we consider only one term in this sum, say the $i$-th. We write $\operatorname{id}_{\Gamma}$ and $v$ for the scalar functions $\left(\operatorname{id}_{\Gamma}\right)_{i}$ and $\left(\mathbf{v}_{H}\right)_{i}$, respectively. We write $\operatorname{id}_{\Gamma_{h}}$ for $\left(\operatorname{id}_{\Gamma_{h}}\right)_{i}$. Note that

$$
\nabla_{\Gamma} \operatorname{id}_{\Gamma}=\mathbf{P} \nabla \mathrm{id}_{\Gamma}=\mathbf{P} e_{i}, \quad \nabla_{\Gamma_{h}} \mathrm{id}_{\Gamma_{h}}=\mathbf{P}_{h} \nabla \mathrm{id}_{\Gamma_{h}}=\mathbf{P}_{h} e_{i},
$$

with $e_{i}$ the $i$-th basis vector in $\mathbb{R}^{3}$. We introduce scalar versions on the functionals $f_{\Gamma}$ and $f_{\Gamma_{h}}$ defined in (2.9) and (2.3) (without loss of generality we can take $\tau:=1$ ):

$$
g(v):=\int_{\Gamma} \nabla_{\Gamma} \operatorname{id}_{\Gamma} \cdot \nabla_{\Gamma} v d s, \quad g_{h}(v):=\int_{\Gamma_{h}} \nabla_{\Gamma_{h}} \operatorname{id}_{\Gamma_{h}} \cdot \nabla_{\Gamma_{h}} v d s .
$$


As noted in Remark 2, $g$ is a bounded linear functional on $H^{1}(U)$. To guarantee that $g_{h}$ and the extension operator in (3.1) are well-defined we assume $v \in H^{1}\left(\Gamma_{h}\right) \cap C\left(\Gamma_{h}\right)$. Therefore, in the analysis in this section we use the function space $W:=H^{1}(U) \cap$ $H^{1}\left(\Gamma_{h}\right) \cap C\left(\Gamma_{h}\right)$.

REMARK 4. If we use a Hood-Taylor pair $\mathbf{V}_{H} \times Q_{H}$ in the discretization of the Navier-Stokes equations, then the $i$-th component $v \in V_{H}$ of $\mathbf{v}_{H} \in \mathbf{V}_{H}=\left(V_{H}\right)^{3}$ is continuous and piecewise polynomial (on the tetrahedral triangulation $\mathcal{S}$ ). Thus $v \in W$ holds.

In this section we first derive, for $v \in W$, a bound for $\left|g(v)-g_{h}(v)\right|$ in terms of $\|v\|_{1, U}:=\|v\|_{H^{1}(U)}$ and $\left\|\nabla_{\Gamma_{h}} v\right\|_{L^{2}\left(\Gamma_{h}\right)}$. This bound is given in Corollary 4.4. Using this bound we then derive a bound for

$$
\sup _{v \in V_{H}} \frac{g(v)-g_{h}(v)}{\|v\|_{1}}
$$

cf. Theorem 4.7. This immediately implies a bound for the approximation error as in (2.8), cf. Corollary 4.8.

The analysis is based on the following splitting

$$
\begin{aligned}
& g(v)-g_{h}(v) \\
& =\int_{\Gamma} \nabla_{\Gamma} \mathrm{id}_{\Gamma} \cdot \nabla_{\Gamma} v d s-\int_{\Gamma_{h}} \nabla_{\Gamma_{h}} \mathrm{id}_{\Gamma}^{e} \cdot \nabla_{\Gamma_{h}} v d s+\int_{\Gamma_{h}} \nabla_{\Gamma_{h}}\left(\mathrm{id}_{\Gamma}^{e}-\mathrm{id}_{\Gamma_{h}}\right) \cdot \nabla_{\Gamma_{h}} v d s \\
& \stackrel{(3.6)}{=} \int_{\Gamma} \nabla_{\Gamma} \mathrm{id}_{\Gamma} \cdot \nabla_{\Gamma} v d s-\int_{\Gamma} \mathbf{A}_{\Gamma_{h}}^{e} \nabla_{\Gamma} \mathrm{id}_{\Gamma} \cdot \nabla_{\Gamma} v_{\Gamma_{h}}^{e} d s+\int_{\Gamma_{h}} \nabla_{\Gamma_{h}}\left(\mathrm{id}_{\Gamma}^{e}-\mathrm{id}_{\Gamma_{h}}\right) \cdot \nabla_{\Gamma_{h}} v d s \\
& =\int_{\Gamma} \nabla_{\Gamma} \mathrm{id}_{\Gamma} \cdot \nabla_{\Gamma}\left(v-v_{\Gamma_{h}}^{e}\right) d s+\int_{\Gamma}\left(\mathbf{I}-\mathbf{A}_{\Gamma_{h}}^{e}\right) \nabla_{\Gamma} \mathrm{id}_{\Gamma} \cdot \nabla_{\Gamma} v_{\Gamma_{h}}^{e} d s \\
& \quad+\int_{\Gamma_{h}} \nabla_{\Gamma_{h}}\left(\mathrm{id}_{\Gamma}^{e}-\mathrm{id}_{\Gamma_{h}}\right) \cdot \nabla_{\Gamma_{h}} v d s .
\end{aligned}
$$

In the lemmas below we derive bounds for the three terms in (4.1). Note that the first two terms do not involve $\operatorname{id}_{\Gamma_{h}}$.

LEMMA 4.1. The following holds:

$$
\left|\int_{\Gamma}\left(\mathbf{I}-\mathbf{A}_{\Gamma_{h}}^{e}\right) \nabla_{\Gamma} \operatorname{id}_{\Gamma} \cdot \nabla_{\Gamma} v_{\Gamma_{h}}^{e} d s\right| \leq c h^{2}\left\|\nabla_{\Gamma_{h}} v\right\|_{L^{2}\left(\Gamma_{h}\right)} \quad \text { for all } \quad v \in W .
$$

Proof. Using the Cauchy-Schwarz inequality and the results in Lemma 3.3, Lemma 3.4 we obtain

$$
\begin{aligned}
& \left|\int_{\Gamma}\left(\mathbf{I}-\mathbf{A}_{\Gamma_{h}}^{e}\right) \nabla_{\Gamma} \operatorname{id}_{\Gamma} \cdot \nabla_{\Gamma} v_{\Gamma_{h}}^{e} d s\right|=\left|\int_{\Gamma}\left(\mathbf{I}-\mathbf{A}_{\Gamma_{h}}^{e}\right) \mathbf{P} \nabla_{\Gamma} \operatorname{id}_{\Gamma} \cdot \nabla_{\Gamma} v_{\Gamma_{h}}^{e} d s\right| \\
& \leq \operatorname{ess}_{\sup _{y \in \Gamma}}\left\|\left(\mathbf{I}-\mathbf{A}_{\Gamma_{h}}^{e}(y)\right) \mathbf{P}(y)\right\|\left\|\nabla_{\Gamma} \operatorname{id}_{\Gamma}\right\|_{L^{2}(\Gamma)}\left\|\nabla_{\Gamma} v_{\Gamma_{h}}^{e}\right\|_{L^{2}(\Gamma)} \\
& \leq c h^{2}\left\|\nabla_{\Gamma_{h}} v\right\|_{L^{2}\left(\Gamma_{h}\right)},
\end{aligned}
$$

and thus the result holds.

LEMMA 4.2. The following holds:

$$
\left|\int_{\Gamma_{h}} \nabla_{\Gamma_{h}}\left(\mathrm{id}_{\Gamma}^{e}-\mathrm{id}_{\Gamma_{h}}\right) \cdot \nabla_{\Gamma_{h}} v d s\right| \leq c h\left\|\nabla_{\Gamma_{h}} v\right\|_{L^{2}\left(\Gamma_{h}\right)} \quad \text { for all } v \in W .
$$


Proof. ¿From Lemma 3.1 we get for $x \in \Gamma_{h}$ (not on an edge),

$$
\begin{aligned}
\nabla_{\Gamma_{h}} \operatorname{id}_{\Gamma}^{e}(x) & =\mathbf{P}_{h}(x)(\mathbf{I}-d(x) \mathbf{H}(x)) \mathbf{P}(x) \nabla_{\Gamma} \operatorname{id}_{\Gamma}(\mathbf{p}(x)) \\
& =\mathbf{P}_{h}(x)(\mathbf{I}-d(x) \mathbf{H}(x)) \mathbf{P}(x) e_{i} .
\end{aligned}
$$

We also have $\nabla_{\Gamma_{h}} \operatorname{id}_{\Gamma_{h}}=\mathbf{P}_{h} \nabla \operatorname{id}_{\Gamma_{h}}=\mathbf{P}_{h} e_{i}$. Hence,

$$
\begin{aligned}
& \left|\int_{\Gamma_{h}} \nabla_{\Gamma_{h}}\left(\mathrm{id}_{\Gamma}^{e}-\mathrm{id}_{\Gamma_{h}}\right) \cdot \nabla_{\Gamma_{h}} v d s\right| \\
& =\left|\int_{\Gamma_{h}}\left(\mathbf{P}_{h}(\mathbf{I}-d \mathbf{H}) \mathbf{P} e_{i}-\mathbf{P}_{h} e_{i}\right) \cdot \nabla_{\Gamma_{h}} v d s\right| \\
& \leq c \operatorname{ess} \sup _{x \in \Gamma_{h}}\left\|\mathbf{P}_{h}(x)(\mathbf{I}-d(x) \mathbf{H}(x)) \mathbf{P}(x)-\mathbf{P}_{h}(x)\right\|\left\|\nabla_{\Gamma_{h}} v\right\|_{L^{2}\left(\Gamma_{h}\right)} \\
& \leq c \operatorname{ess} \sup _{x \in \Gamma_{h}}\left(\left\|\mathbf{P}_{h}(x) \mathbf{P}(x)-\mathbf{P}_{h}(x)\right\|\right. \\
& \left.+|d(x)|\left\|\mathbf{P}_{h}(x) \mathbf{H}(x) \mathbf{P}(x)\right\|\right)\left\|\nabla_{\Gamma_{h}} v\right\|_{L^{2}\left(\Gamma_{h}\right)} .
\end{aligned}
$$

Note that $|d(x)| \leq c h^{2}$ for $x \in \Gamma_{h}$, and

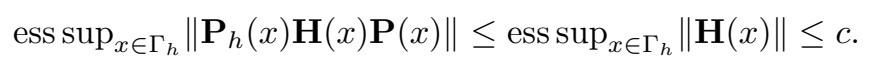

For the term in (4.3) we have (we drop $x$ in the notation):

$$
\left\|\mathbf{P}_{h} \mathbf{P}-\mathbf{P}_{h}\right\|=\left\|\mathbf{P}_{h} \mathbf{n n}^{T}\right\| \leq\left\|\mathbf{P}_{h} \mathbf{n}\right\| \leq\left\|\mathbf{P}_{h} \mathbf{n}+\mathbf{P n}_{h}\right\|+\left\|\mathbf{P} \mathbf{n}_{h}\right\| .
$$

For the first term we get, using (3.7),

$$
\left\|\mathbf{P}_{h} \mathbf{n}+\mathbf{P} \mathbf{n}_{h}\right\|=\left\|\left(1-\mathbf{n}^{T} \mathbf{n}_{h}\right)\left(\mathbf{n}+\mathbf{n}_{h}\right)\right\| \leq 2\left|1-\mathbf{n}^{T} \mathbf{n}_{h}\right| \leq c h^{2} .
$$

¿From (2.6) we get $\left\|\mathbf{P n}_{h}\right\| \leq c h$ (a.e. on $\Gamma_{h}$ ). Thus $\left\|\mathbf{P}_{h}(x) \mathbf{P}(x)-\mathbf{P}_{h}(x)\right\| \leq c h$ holds a.e. on $\Gamma_{h}$. As an upper bound for (4.2) we obtain $c h\left\|\nabla_{\Gamma_{h}} v\right\|_{L^{2}\left(\Gamma_{h}\right)}$. $\mathbf{Q}$

LEMMA 4.3. The following holds:

$$
\left|\int_{\Gamma} \nabla_{\Gamma} \operatorname{id}_{\Gamma} \cdot \nabla_{\Gamma}\left(v-v_{\Gamma_{h}}^{e}\right) d s\right| \leq c h\|v\|_{1, U} \quad \text { for all } \quad v \in W .
$$

Proof. We take $v \in C^{1}(U)$. For $y \in \Gamma$ we have $v_{\Gamma_{h}}^{e}(y)=v(y \pm \delta(y) \mathbf{n}(y))$ with a unique $\delta(y) \geq 0$ such that $y \pm \delta(y) \mathbf{n}(y) \in \Gamma_{h}$. Note that $\delta(y) \leq c h^{2}$ holds. Let $U_{m} \subset U$ be a strip around $\Gamma$ that contains $\Gamma_{h}$ and has width $m \leq c h^{2}$. We now have

$$
\begin{aligned}
\left|\int_{\Gamma} \nabla_{\Gamma} \operatorname{id}_{\Gamma} \cdot \nabla_{\Gamma}\left(v-v_{\Gamma_{h}}^{e}\right) d s(y)\right| & =\left|\int_{\Gamma} \Delta_{\Gamma} \operatorname{id}_{\Gamma}(v(y)-v(y \pm \delta(y) \mathbf{n}(y))) d s(y)\right| \\
& \leq \int_{\Gamma}\left|\Delta_{\Gamma} \operatorname{id}_{\Gamma}\right|\left|\int_{0}^{\delta(y)} \frac{\partial v}{\partial t}(y \pm t \mathbf{n}(y)) d t\right| d s(y) \\
& \leq c \int_{\Gamma} \int_{0}^{\delta(y)}\left|\frac{\partial v}{\partial t}(y \pm t \mathbf{n}(y))\right| d t d s(y) .
\end{aligned}
$$

For $x=y \pm t \mathbf{n}(y)$ with $0 \leq t \leq \delta(y)$ we use $\mathbf{n}(x)=\mathbf{n}(\mathbf{p}(x))=\mathbf{n}(y)$ and obtain

$$
\begin{aligned}
\left|\int_{\Gamma} \nabla_{\Gamma} \operatorname{id}_{\Gamma} \cdot \nabla_{\Gamma}\left(v-v_{\Gamma_{h}}^{e}\right) d s(y)\right| & \leq c \int_{U_{m}}|\mathbf{n}(x) \cdot \nabla v(x)| d x \\
& \leq c\left(\int_{U_{m}} 1 d x\right)^{\frac{1}{2}}\left(\int_{U_{m}}(\nabla v)^{2} d x\right)^{\frac{1}{2}} \leq c h\|v\|_{1, U} .
\end{aligned}
$$


A density argument yields the same bound for all $v \in W$. $\mathrm{\square}$

A direct consequence of the previous three lemmas is the following corollary.

Corollary 4.4. The three terms in (4.1) can be bounded by

$$
\begin{aligned}
& \left|\int_{\Gamma} \nabla_{\Gamma} \operatorname{id}_{\Gamma} \cdot \nabla_{\Gamma}\left(v-v_{\Gamma_{h}}^{e}\right) d s\right| \leq c h\|v\|_{1, U}, \\
& \left|\int_{\Gamma}\left(\mathbf{I}-\mathbf{A}_{\Gamma_{h}}^{e}\right) \nabla_{\Gamma} \operatorname{id}_{\Gamma} \cdot \nabla_{\Gamma} v_{\Gamma_{h}}^{e} d s\right| \leq c h^{2}\left\|\nabla_{\Gamma_{h}} v\right\|_{L^{2}\left(\Gamma_{h}\right)}, \\
& \left|\int_{\Gamma_{h}} \nabla_{\Gamma_{h}}\left(\operatorname{id}_{\Gamma}^{e}-\operatorname{id}_{\Gamma_{h}}\right) \cdot \nabla_{\Gamma_{h}} v d s\right| \leq c h\left\|\nabla_{\Gamma_{h}} v\right\|_{L^{2}\left(\Gamma_{h}\right)},
\end{aligned}
$$

and thus

$$
\left|g(v)-g_{h}(v)\right| \leq c h\|v\|_{1, U}+c h^{2}\left\|\nabla_{\Gamma_{h}} v\right\|_{L^{2}\left(\Gamma_{h}\right)}+c h\left\|\nabla_{\Gamma_{h}} v\right\|_{L^{2}\left(\Gamma_{h}\right)} \quad \text { for all } v \in W
$$

holds.

In view of Corollary 4.4 and the error measure in (2.8) we want to derive a bound for $\left\|\nabla_{\Gamma_{h}} v\right\|_{L^{2}\left(\Gamma_{h}\right)}$ in terms of $\|v\|_{1}$ for $v$ from the scalar finite element space $V_{H}$. An obvious approach is to apply an inverse inequality combined with a trace theorem, resulting in:

$$
\left\|\nabla_{\Gamma_{h}} v\right\|_{L^{2}\left(\Gamma_{h}\right)} \leq c h_{\min }^{-1}\|v\|_{L^{2}\left(\Gamma_{h}\right)} \leq c h_{\min }^{-1}\|v\|_{1} \quad \text { for all } v \in V_{H} .
$$

This, however, is too crude (cf. the bound in Corollary 4.4). In order to be able to derive a better bound than the one in (4.8) we have to introduce some further assumptions related to the family of triangulations $\left\{\Gamma_{h}\right\}_{h>0}$. This family is assumed to be shape-regular, i.e., all angles in of all triangles are uniformly (w.r.t. $h$ ) bounded from below, but not necessarily quasi-uniform. We assume that to each triangulation $\Gamma_{h}=\cup_{T \in \mathcal{F}_{h}} T$ there can be associated a set of tetrahedra $\mathcal{S}_{h}$ with the following properties:

For each $T \in \mathcal{F}_{h}$ there is a corresponding $S_{T} \in \mathcal{S}_{h}$ with $T \subset S_{T}$.

For $T_{1}, T_{2} \in \mathcal{F}_{h}$ with $T_{1} \neq T_{2}$ we have $\operatorname{meas}_{3}\left(S_{T_{1}} \cap S_{T_{2}}\right)=0$.

The family $\left\{\mathcal{S}_{h}\right\}_{h>0}$ is shape-regular.

$c_{0} h \leq \operatorname{diam}\left(S_{T}\right) \leq c h$ for all $T \in \mathcal{F}_{h}$, with $c_{0}>0$ (quasi-uniformity).

For each $S_{T} \in \mathcal{S}_{h}$ there is a tetrahedron $S \in \mathcal{S}$ such that $S_{T} \subset S$.

Recall that $\mathcal{S}$ is the (fixed) tetrahedral triangulation that is used in the finite element discretization of the Navier-Stokes problem in (1.6). Note that the set of tetrahedra $\mathcal{S}_{h}$ has to be defined only close to the approximate interface $\Gamma_{h}$ and that this set not necessarily forms a regular tetrahedral triangulation.

REMARK 5. Consider the construction of $\left\{\Gamma_{h}\right\}_{h>0}$ as in Remark 3. The approximate interface $\Gamma_{h}$ is the zero level of the function $I\left(d_{h}\right)$, which is continuous piecewise linear on the tetrahedral triangulation $\mathcal{T}^{\prime}$ :

$$
\Gamma_{h}=\cup_{T} T
$$

Each $T$ is a triangle or a quadrilateral. To each $T$ there can be associated a tetrahedron $S_{T} \in \mathcal{T}^{\prime}$ such that $T \subset S_{T}$. If $T$ is a quadrilateral then we can subdivide $T$ and $S_{T}$ in two disjoint triangles $T_{1}, T_{2}$ and two disjoint tetrahedra $S_{T_{1}}, S_{T_{2}}$, respectively, 
such that $T_{i} \subset S_{T_{i}} \subset S_{T}$ for $i=1,2$. One can check that this construction results in a family $\left\{\mathcal{S}_{h}\right\}_{h>0}$ that satisfies the conditions (4.9)-(4.13).

We note that the construction of the approximate interface does not guarantee that the family $\left\{\Gamma_{h}\right\}_{h>0}$ is shape-regular. In practice relatively few triangles with small interior angles are likely to occur, which, however in our experience does not lead to a significant deterioration of the approximation quality.

In the following lemma we consider a standard affine mapping between a tetrahedron $S_{T} \in \mathcal{S}_{h}$ and the reference unit tetrahedron and apply it to the triangle $T \subset S_{T}$.

LEMma 4.5. Assume that the family $\left\{\Gamma_{h}\right\}_{h>0}$ is shape-regular and that for the associated family of sets of tetrahedra $\left\{\mathcal{S}_{h}\right\}_{h>0}$ the conditions (4.9)-(4.13) are satisfied. Take $T \in \mathcal{F}_{h}$ and the corresponding $S_{T} \in \mathcal{S}_{h}$. Let $\hat{S}$ be the reference unit tetrahedron and $F(x)=\mathbf{J} x+\mathbf{b}$ be an affine mapping such that $F(\hat{S})=S_{T}$. Define $\hat{T}:=F^{-1}(T)$. The following holds:

$$
\begin{gathered}
\|\mathbf{J}\|^{2} \frac{\operatorname{meas}_{3}(\hat{S})}{\operatorname{meas}_{3}\left(S_{T}\right)} \leq c h^{-1}, \\
\left\|\mathbf{J}^{-1}\right\|^{2} \frac{\operatorname{meas}_{2}(T)}{\operatorname{meas}_{2}(\hat{T})} \leq c
\end{gathered}
$$

with constants $c$ independent of $T$ and $h$.

Proof. Let $\rho\left(S_{T}\right)$ be the diameter of the maximal ball contained in $S_{T}$ and similarly for $\rho(\hat{S})$. From standard finite element theory we have

$$
\|\mathbf{J}\| \leq \frac{\operatorname{diam}\left(S_{T}\right)}{\rho(\hat{S})}, \quad\left\|\mathbf{J}^{-1}\right\| \leq \frac{\operatorname{diam}(\hat{S})}{\rho\left(S_{T}\right)} .
$$

Using (4.11) and (4.12) we then get

$$
\|\mathbf{J}\|^{2} \frac{\operatorname{meas}_{3}(\hat{S})}{\operatorname{meas}_{3}\left(S_{T}\right)} \leq c \frac{\operatorname{diam}\left(S_{T}\right)^{2}}{\operatorname{meas}_{3}\left(S_{T}\right)} \leq c \operatorname{diam}\left(S_{T}\right)^{-1} \leq c h^{-1},
$$

and thus the result in (4.14) holds.

The vertices of $\hat{T}=F^{-1}(T)$ are denoted by $\hat{V}_{i}, i=1,2,3$. Let $\hat{V}_{1} \hat{V}_{2}$ be a longest edge of $\hat{T}$ and $\hat{M}$ the point on this edge such that $\hat{M} \hat{V}_{3}$ is perpendicular to $\hat{V}_{1} \hat{V}_{2}$. Define $V_{i}:=F\left(\hat{V}_{i}\right), i=1,2,3$, and $M:=F(\hat{M})$. Then $V_{i}, i=1,2,3$, are the vertices of $T$ and $M$ lies on the edge $V_{1} V_{2}$. Using the fact that $T$ is an element of a shape-regular family we get

$$
\begin{aligned}
\operatorname{meas}_{2}(\hat{T}) & =\frac{1}{2}\left\|\hat{V}_{1}-\hat{V}_{2}\right\|\left\|\hat{V}_{3}-\hat{M}\right\|=\frac{1}{2}\left\|\mathbf{J}^{-1}\left(V_{1}-V_{2}\right)\right\|\left\|\mathbf{J}^{-1}\left(V_{3}-M\right)\right\| \\
& \geq \frac{1}{2}\|\mathbf{J}\|^{-2}\left\|V_{1}-V_{2}\right\|\left\|V_{3}-M\right\| \geq c \frac{\rho(\hat{S})^{2}}{\operatorname{diam}\left(S_{T}\right)^{2}} \operatorname{meas}_{2}(\mathrm{~T}),
\end{aligned}
$$

with a constant $c>0$. Thus we obtain

$$
\left\|\mathbf{J}^{-1}\right\|^{2} \frac{\operatorname{meas}_{2}(T)}{\operatorname{meas}_{2}(\hat{T})} \leq c \frac{\operatorname{diam}(\hat{S})^{2}}{\rho\left(S_{T}\right)^{2}} \frac{\operatorname{diam}\left(S_{T}\right)^{2}}{\rho(\hat{S})^{2}} \leq c,
$$

which completes the proof. 
THEOREM 4.6. Assume that the family $\left\{\Gamma_{h}\right\}_{h>0}$ is shape-regular and that for the associated family of sets of tetrahedra $\left\{\mathcal{S}_{h}\right\}_{h>0}$ the conditions (4.9)-(4.13) are satisfied. The following holds:

$$
\left\|\nabla_{\Gamma_{h}} v\right\|_{L^{2}\left(\Gamma_{h}\right)} \leq c h^{-\frac{1}{2}}\|v\|_{1} \quad \text { for all } v \in V_{H}
$$

Proof. Note that

$$
\left\|\nabla_{\Gamma_{h}} v\right\|_{L^{2}\left(\Gamma_{h}\right)}^{2}=\sum_{T \in \mathcal{F}_{h}}\left\|\nabla_{T} v\right\|_{L^{2}(T)}^{2}
$$

Take $T \in \mathcal{F}_{h}$ and let $S_{T}$ be the associated tetrahedron as explained above. Let $\hat{S}$ be the reference unit tetrahedron and $F: \hat{S} \rightarrow S_{T}$ as in Lemma 4.5. Define $\hat{v}:=v \circ F$. Using standard transformation rules and Lemma 4.5 we get

$$
\begin{aligned}
\left\|\nabla_{T} v\right\|_{L^{2}(T)}^{2} & =\left\|\mathbf{P}_{h} \nabla v\right\|_{L^{2}(T)}^{2} \leq\|\nabla v\|_{L^{2}(T)}^{2}=\sum_{|\alpha|=1}\left\|\partial^{\alpha} v\right\|_{L^{2}(T)}^{2} \\
& \leq c\left\|\mathbf{J}^{-1}\right\|^{2} \sum_{|\alpha|=1}\left\|\left(\partial^{\alpha} \hat{v}\right) \circ F^{-1}\right\|_{L^{2}(T)}^{2} \\
& \leq c\left\|\mathbf{J}^{-1}\right\|^{2} \frac{\operatorname{meas}_{2}(T)}{\operatorname{meas}_{2}(\hat{T})} \sum_{|\alpha|=1}\left\|\partial^{\alpha} \hat{v}\right\|_{L^{2}(\hat{T})}^{2} \leq c \sum_{|\alpha|=1}\left\|\partial^{\alpha} \hat{v}\right\|_{L^{2}(\hat{T})}^{2} \\
& \leq c \sum_{|\alpha|=1} \max _{x \in \hat{T}}\left|\partial^{\alpha} \hat{v}(x)\right|^{2} \leq c \sum_{|\alpha|=1} \max _{x \in \hat{S}}\left|\partial^{\alpha} \hat{v}(x)\right|^{2},
\end{aligned}
$$

with a constant $c$ independent of $T$. From (4.13) it follows that $\hat{v}$ is a polynomial on $\hat{S}$ of maximal degree $k$, where $k$ depends only on the choice of the finite element space $\mathbf{V}_{H}$. On $P_{k}^{*}:=\left\{p \in P_{k} \mid p(0)=0\right\}$ we have, due to equivalence of norms:

$$
\sum_{|\alpha|=1} \max _{x \in \hat{S}}\left|\partial^{\alpha} \hat{v}(x)\right|^{2} \leq c \sum_{|\alpha|=1}\left\|\partial^{\alpha} \hat{v}\right\|_{L^{2}(\hat{S})}^{2} \quad \text { for all } \hat{v} \in P_{k}^{*}
$$

Because, for $\hat{v} \in P_{k}$ and $|\alpha|=1, \partial^{\alpha} \hat{v}$ is independent of $\hat{v}(0)$, the same inequality holds for all $\hat{v} \in P_{k}$. Thus we get

$$
\begin{aligned}
\left\|\nabla_{T} v\right\|_{L^{2}(T)}^{2} & \leq c \sum_{|\alpha|=1}\left\|\partial^{\alpha} \hat{v}\right\|_{L^{2}(\hat{S})}^{2} \leq c\|\mathbf{J}\|^{2} \sum_{|\alpha|=1}\left\|\left(\partial^{\alpha} v\right) \circ F\right\|_{L^{2}(\hat{S})}^{2} \\
& =c\|\mathbf{J}\|^{2} \frac{\operatorname{meas}_{3}(\hat{S})}{\operatorname{meas}_{3}\left(S_{T}\right)} \sum_{|\alpha|=1}\left\|\partial^{\alpha} v\right\|_{L^{2}\left(S_{T}\right)}^{2} \leq c h^{-1}\|\nabla v\|_{L^{2}\left(S_{T}\right)}^{2},
\end{aligned}
$$

with a constant $c$ independent of $T$ and $h$. Using (4.10) we finally obtain

$$
\begin{aligned}
\left\|\nabla_{\Gamma_{h}} v\right\|_{L^{2}\left(\Gamma_{h}\right)}^{2} & \leq c h^{-1} \sum_{T \in \mathcal{F}_{h}}\|\nabla v\|_{L^{2}\left(S_{T}\right)}^{2} \\
& \leq c h^{-1} \int_{\Omega}(\nabla v)^{2} d x \leq c h^{-1}\|v\|_{1}^{2},
\end{aligned}
$$

which proves the result.

We now present the main result of this paper. 
TheOREM 4.7. Let the assumptions be as in Theorem 4.6. The following holds:

$$
\sup _{v \in V_{H}} \frac{g(v)-g_{h}(v)}{\|v\|_{1}} \leq c \sqrt{h}
$$

Proof. Combine the result in Corollary 4.4 with the one in Theorem 4.6.

As a direct consequence we obtain:

Corollary 4.8. Let the assumptions be as in Theorem 4.6. For $f_{\Gamma}$ and $f_{\Gamma_{h}}$ as defined in section 2 the following holds:

$$
\sup _{\mathbf{v} \in \mathbf{V}_{H}} \frac{f_{\Gamma}\left(\mathbf{v}_{H}\right)-f_{\Gamma_{h}}\left(\mathbf{v}_{H}\right)}{\left\|\mathbf{v}_{H}\right\|_{1}} \leq \tau c \sqrt{h} .
$$

Proof. Note that

$f_{\Gamma}\left(\mathbf{v}_{H}\right)-f_{\Gamma_{h}}\left(\mathbf{v}_{H}\right)=\tau \sum_{i=1}^{3}\left(\int_{\Gamma} \nabla_{\Gamma}\left(\operatorname{id}_{\Gamma}\right)_{i} \cdot \nabla_{\Gamma}\left(\mathbf{v}_{H}\right)_{i} d s-\int_{\Gamma_{h}} \nabla_{\Gamma_{h}}\left(\operatorname{id}_{\Gamma_{h}}\right)_{i} \cdot \nabla_{\Gamma_{h}}\left(\mathbf{v}_{H}\right)_{i} d s\right)$,

and use the result in Theorem 4.7. $\mathrm{a}$

An upper bound $\mathcal{O}(\sqrt{h})$ as in Corollary 4.8 for the error in the approximation of the localized force term may seem rather pessimistic, beause $\Gamma_{h}$ is an $\mathcal{O}\left(h^{2}\right)$ accurate approximation of $\Gamma$. Numerical experiments in section 6 , however, indicate that the bound is sharp.

5. Improved approximation of the localized force term $f_{\Gamma}\left(\mathbf{v}_{h}\right)$. In this section we show how the approximation of the localized force term can be improved, resulting in an improved error bound of the form $\mathcal{O}(h)$ (instead of $\mathcal{O}(\sqrt{h})$ ).

¿From Corollary 4.4 and Theorem 4.6 we see that the $\sqrt{h}$ behaviour is caused by the estimate in (4.7):

$$
\left|\int_{\Gamma_{h}} \nabla_{\Gamma_{h}}\left(\operatorname{id}_{\Gamma}^{e}-\operatorname{id}_{\Gamma_{h}}\right) \cdot \nabla_{\Gamma_{h}} v d s\right| \leq c h\left\|\nabla_{\Gamma_{h}} v\right\|_{L^{2}\left(\Gamma_{h}\right)} .
$$

The term $\nabla_{\Gamma_{h}} \mathrm{id}_{\Gamma_{h}}$ that is used in $g_{h}(v)$ occurs in (5.1) but not in the other two terms of the splitting, cf. (4.5), (4.6). We consider

$$
\tilde{g}_{h}(v)=\int_{\Gamma_{h}} m_{h} \cdot \nabla_{\Gamma_{h}} v d s
$$

and try to find a function $m_{h}=m_{h}(x)$ such that $\tilde{g}_{h}(v)$ remains easily computable and the bound in (5.1) is improved if we use $m_{h}$ instead of $\nabla_{\Gamma_{h}} \mathrm{id}_{\Gamma_{h}}$. The latter condition is trivially satisfied for $m_{h}=\nabla_{\Gamma_{h}}$ id ${ }_{\Gamma}^{e}$ (leading to a bound 0 in (5.1)). This choice, however, does not satisfy the first condition, because $\Gamma$ is not known. We now discuss another possibility, that is used in the experiments in section 6 .

Due to $|d(x)| \leq c h^{2}$ we get from Lemma 3.1, for $x \in \Gamma_{h}$ :

$$
\nabla_{\Gamma_{h}} \operatorname{id}_{\Gamma}^{e}(x)=\mathbf{P}_{h}(x) \mathbf{P}(x) \nabla_{\Gamma} \operatorname{id}_{\Gamma}(\mathbf{p}(x))+\mathcal{O}\left(h^{2}\right)=\mathbf{P}_{h}(x) \mathbf{P}(x) e_{i}+\mathcal{O}\left(h^{2}\right) .
$$

In the construction of the interface $\Gamma_{h}$, cf. Remark 3 , we have a piecewise quadratic function $d_{h} \approx d$ available. Define

$$
\tilde{\mathbf{n}}_{h}(x):=\frac{\nabla d_{h}(x)}{\left\|\nabla d_{h}(x)\right\|}, \quad \tilde{\mathbf{P}}_{h}(x) ;=\mathbf{I}-\tilde{\mathbf{n}}_{h}(x) \tilde{\mathbf{n}}_{h}(x)^{T}, \quad x \in \Gamma_{h} .
$$


Thus an obvious modification is based on the choice $m_{h}(x)=\mathbf{P}_{h}(x) \tilde{\mathbf{P}}_{h}(x) e_{i}$, i.e.,

$$
\tilde{g}_{h}(v):=\int_{\Gamma_{h}} \mathbf{P}_{h}(x) \tilde{\mathbf{P}}_{h}(x) e_{i} \cdot \nabla_{\Gamma_{h}} v d s
$$

In this approach the approximate interface $\Gamma_{h}$ is not changed (piecewise planar). For piecewise quadratics $d_{h}$ and $v$, the function $\nabla_{\Gamma_{h}} v=P_{h} \nabla v$ is piecewise linear and $\mathbf{P}_{h} \tilde{\mathbf{P}}_{h} e_{i}$ is piecewise (very) smooth on the segments of $\Gamma_{h}$. Hence, the functional in (5.2) can be evaluated easily.

Under reasonable assumptions the modified functional indeed yields a better error bound:

LEMMA 5.1. Assume that there exists $p>0$ such that

$$
\left\|\nabla d_{h}(x)-\nabla d(x)\right\| \leq c h^{p}, \quad \text { for } \quad x \in \Gamma_{h} .
$$

Then the following holds:

$$
\left|\int_{\Gamma_{h}}\left(\nabla_{\Gamma_{h}} \operatorname{id}_{\Gamma}^{e}-\mathbf{P}_{h} \tilde{\mathbf{P}}_{h} e_{i}\right) \cdot \nabla_{\Gamma_{h}} v d s\right| \leq c h^{\min \{p, 2\}}\left\|\nabla_{\Gamma_{h}} v\right\|_{L^{2}\left(\Gamma_{h}\right)} \quad \text { for all } v \in W
$$

Proof. Using $\|\nabla d\|=1$ it follows that $\left\|\nabla d_{h}\right\|=1+\mathcal{O}\left(h^{p}\right)$ holds. We can use the line of reasoning as in the proof of Lemma 4.2. The term in (4.4) remains the same. Instead of the term in (4.3) we now get $\left\|\mathbf{P}_{h}(x) \mathbf{P}(x)-\mathbf{P}_{h}(x) \tilde{\mathbf{P}}_{h}(x)\right\|$. We drop $x$ in the notation and using the assumption we obtain

$$
\begin{aligned}
\left\|\mathbf{P}_{h} \mathbf{P}-\mathbf{P}_{h} \tilde{\mathbf{P}}_{h}\right\| & =\left\|\mathbf{P}_{h}\left(\mathbf{P}-\tilde{\mathbf{P}}_{h}\right)\right\| \leq\left\|\mathbf{n} \mathbf{n}^{T}-\tilde{\mathbf{n}}_{h} \tilde{\mathbf{n}}_{h}^{T}\right\| \\
& \leq\left\|\left(\mathbf{n}-\tilde{\mathbf{n}}_{h}\right) \mathbf{n}^{T}\right\|+\left\|\tilde{\mathbf{n}}_{h}\left(\mathbf{n}-\tilde{\mathbf{n}}_{h}\right)^{T}\right\|=2\left\|\mathbf{n}-\tilde{\mathbf{n}}_{h}\right\| \\
& =2\left\|\nabla d-\frac{\nabla d_{h}}{\left\|\nabla d_{h}\right\|}\right\| \\
& \leq 2\left|1-\left\|\nabla d_{h}\right\|^{-1}\right|\left\|\nabla d_{h}\right\|+2\left\|\nabla d-\nabla d_{h}\right\| \leq c h^{p} .
\end{aligned}
$$

Thus we get an estimate $\left\|\mathbf{P}_{h} \mathbf{P}-\mathbf{P}_{h} \tilde{\mathbf{P}}_{h}\right\| \leq c h^{p}$. Combined with the inequality $|d(x)|\left\|\mathbf{P}_{h}(x) \mathbf{H}(x) \mathbf{P}(x)\right\| \leq c h^{2}$ for the term in (4.4) this proves the result.

If we assume that the condition in (5.3) is satisfied for $p=2$, which is reasonable for a piecewise quadratic approximation $d_{h}$ of $d$, we get the following improvement due to the modified functional $\tilde{g}_{h}$, cf. Corollary 4.4 :

$$
\left|g(v)-\tilde{g}_{h}(v)\right| \leq c h\|v\|_{1, U}+c h^{2}\left\|\nabla_{\Gamma_{h}} v\right\|_{L^{2}\left(\Gamma_{h}\right)} \quad \text { for all } v \in W .
$$

Combining this with the result in Theorem 4.6 yields (under the assumption as in Theorem 4.6):

$$
\left|g(v)-\tilde{g}_{h}(v)\right| \leq c h\|v\|_{1, U}+c h^{1 \frac{1}{2}}\|v\|_{1} \quad \text { for all } v \in V_{H}
$$

Hence, using this modified functional $\tilde{g}_{h}$ we have a $\mathcal{O}(h)$ error bound. This significant improvement (compared to the $\mathcal{O}(\sqrt{h})$ error bound for the functional $g_{h}$ ) is confirmed by the numerical experiments in the next section. 


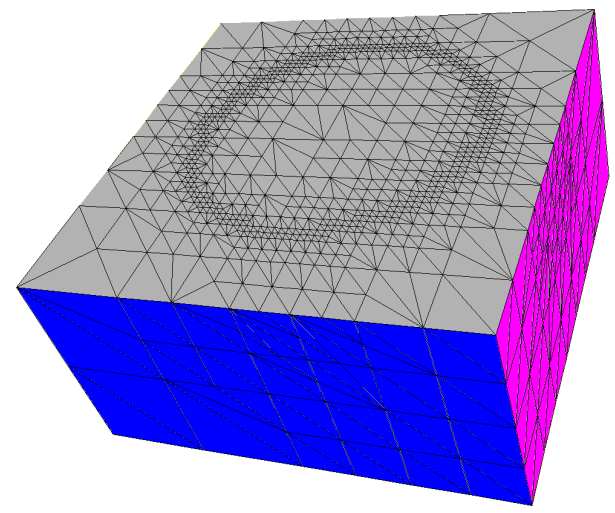

FIG. 6.1. Lower half part of the 4 times refined mesh $\mathcal{T}_{4}$.

6. Numerical experiments. In this section we present results of a numerical experiment which indicates that the $\mathcal{O}(\sqrt{h})$ bound in Corollary 4.8 is sharp. Furthermore, for the improved approximation described in section 5 the $\mathcal{O}(h)$ bound will be confirmed numerically.

We consider the domain $\Omega:=[-1,1]^{3}$ where the ball $\Omega_{1}:=\{\mathbf{x} \in \Omega \mid\|\mathbf{x}\|<R\}$ is located in the center of the domain. In our experiments we take $R=\frac{1}{2}$.

For the discretizaton a uniform tetrahedral mesh $\mathcal{T}_{0}$ is used where the vertices form a $6 \times 6 \times 6$ lattice, hence $h_{0}=\frac{1}{5}$. This coarse mesh $\mathcal{T}_{0}$ is locally refined in the vicinity of $\Gamma=\partial \Omega_{1}$ using an adaptive refinement algorithm presented in [13]. This repeated refinement process yields the gradually refined meshes $\mathcal{T}_{1}, \mathcal{T}_{2}, \ldots$ with local (i.e., close to the interface) mesh sizes $h_{i}=\frac{1}{5} 2^{-i}, i=1,2, \ldots$. Part of the tetrahedral triangulation $\mathcal{T}_{4}$ is shown in figure 6.1. The corresponding finite element spaces $\mathbf{V}_{i}:=$ $\mathbf{V}_{h_{i}}=\left(V_{h_{i}}\right)^{3}$ consists of vector functions where each component is a continuous piecewise quadratic function on $\mathcal{T}_{i}$.

The interface $\Gamma=\partial \Omega_{1}$ is a sphere and thus the curvature $\mathcal{K}=\frac{2}{R}$ is constant. If we discretize the flow problem using $\mathbf{V}_{i}$ as discrete velocity space, we have to approximate the surface tension force

$$
f_{\Gamma}(\mathbf{v})=\frac{2 \tau}{R} \int_{\Gamma} \mathbf{n}_{\Gamma} \cdot \mathbf{v} d s=\tau \int_{\Gamma} \nabla_{\Gamma} \operatorname{id}_{\Gamma} \cdot \nabla_{\Gamma} \mathbf{v} d s, \quad \mathbf{v} \in \mathbf{V}_{i}
$$

To simplify notation, we take a fixed $i \geq 0$ and the corresponding local mesh size parameter is denoted by $h=h_{i}$. For the approximation of the interface we use the following approach (cf. Remark 3). The interface $\Gamma$ is the zero level of the signed distance function $d$. In this test problem $d$ is known. For the finite element approximation $d_{h} \in V_{h}$ of $d$ we take the continuous piecewise quadratic function on $\mathcal{T}_{i}$ that interpolates $d$ at the vertices and midpoints of edges. Then $I\left(d_{h}\right) \in V_{h_{i+1}}$ is the continous piecewise linear function on $\mathcal{T}_{i}^{\prime}$ that interpolates $d_{h}$ at the vertices of all tetrahedra in $\mathcal{T}_{i}^{\prime}$, cf. Remark 3 (note that in this test problem $d_{h}$ can also be computed by piecewise linear interpolation of $d$ on $\mathcal{T}_{i}^{\prime}$ ). The approximation of $\Gamma$ is defined by

$$
\Gamma_{h}=\left\{x \in \Omega \mid I\left(d_{h}\right)(x)=0\right\} .
$$


The discrete approximation of the surface tension force is

$$
f_{\Gamma_{h}}(\mathbf{v})=\tau \int_{\Gamma_{h}} \nabla_{\Gamma_{h}} \operatorname{id}_{\Gamma_{h}} \cdot \nabla_{\Gamma} \mathbf{v} d s, \quad \mathbf{v} \in \mathbf{V}_{i} .
$$

We are interested in, cf. Corollary 4.8,

$$
\left\|f_{\Gamma}-f_{\Gamma_{h}}\right\|_{\mathbf{V}_{i}^{\prime}}:=\sup _{\mathbf{v} \in \mathbf{V}_{i}} \frac{f_{\Gamma}(\mathbf{v})-f_{\Gamma_{h}}(\mathbf{v})}{\|\mathbf{v}\|_{1}} .
$$

The evaluation of $f_{\Gamma}(\mathbf{v})$, for $\mathbf{v} \in \mathbf{V}_{i}$, requires the computation of integrals on curved triangles or quadrilaterals $\Gamma \cap S$ where $S$ is a tetrahedron from the mesh $\mathcal{T}_{i}$. We are not able to compute these exactly. Therefore, we introduce an artificial force term which, in this model problem with a known constant curvature, is computable and sufficiently close to $f_{\Gamma}$.

Lemma 6.1. For $\mathbf{v} \in \mathbf{V}=\left(H_{0}^{1}(\Omega)\right)^{3}$ define

$$
\hat{f}_{\Gamma_{h}}(\mathbf{v}):=\frac{2 \tau}{R} \int_{\Gamma_{h}} \mathbf{n}_{h} \cdot \mathbf{v} d s
$$

$\left(\mathbf{n}_{h}\right.$ : piecewise constant outward unit normal on $\left.\Gamma_{h}\right)$. Then the following inequality holds:

$$
\left\|f_{\Gamma}-\hat{f}_{\Gamma_{h}}\right\|_{\mathbf{V}^{\prime}} \leq c h
$$

Proof. Let $\Omega_{1, h} \subset \Omega$ be the domain enclosed by $\Gamma_{h}$, i.e. $\partial \Omega_{1, h}=\Gamma_{h}$. We define $D_{h}^{+}:=\Omega_{1} \backslash \Omega_{1, h}, D_{h}^{-}:=\Omega_{1, h} \backslash \Omega_{1}$ and $D_{h}:=D_{h}^{+} \cup D_{h}^{-}$. Due to Stokes theorem, for $\mathbf{v} \in \mathbf{V}$ we have

$$
\begin{aligned}
\left|f_{\Gamma}(\mathbf{v})-\hat{f}_{\Gamma_{h}}(\mathbf{v})\right| & =\frac{2 \tau}{R}\left|\int_{\Omega_{1}} \operatorname{div} \mathbf{v} d x-\int_{\Omega_{1, h}} \operatorname{div} \mathbf{v} d x\right| \\
& =\frac{2 \tau}{R}\left|\int_{D_{h}^{+}} \operatorname{div} \mathbf{v} d \mathbf{x}-\int_{D_{h}^{-}} \operatorname{div} \mathbf{v} d x\right| \\
& \leq \frac{2 \tau}{R} \int_{D_{h}}|\operatorname{div} \mathbf{v}| d x
\end{aligned}
$$

Using the Cauchy-Schwarz inequality, we get the estimate

$$
\left|f_{\Gamma}(\mathbf{v})-\hat{f}_{\Gamma_{h}}(\mathbf{v})\right| \leq c \sqrt{\left|D_{h}\right|}\|\mathbf{v}\|_{1} \quad \text { for all } \mathbf{v} \in \mathbf{V}
$$

For the piecewise planar approximation $\Gamma_{h}$ of the interface $\Gamma$ we have $\left|D_{h}\right|=\mathcal{O}\left(h^{2}\right)$ and thus (6.3) holds.

¿From Lemma 6.1 we obtain $\left\|f_{\Gamma}-\hat{f}_{\Gamma_{h}}\right\|_{\mathbf{V}_{j}^{\prime}} \leq c h$ with a constant $c$ independent of $j$. Thus we have

$$
\left\|\hat{f}_{\Gamma_{h}}-f_{\Gamma_{h}}\right\|_{\mathbf{V}_{i}^{\prime}}-c h \leq\left\|f_{\Gamma}-f_{\Gamma_{h}}\right\|_{\mathbf{V}_{i}^{\prime}} \leq\left\|\hat{f}_{\Gamma_{h}}-f_{\Gamma_{h}}\right\|_{\mathbf{V}_{i}^{\prime}}+c h
$$

The term $\left\|\hat{f}_{\Gamma_{h}}-f_{\Gamma_{h}}\right\|_{\mathbf{V}_{i}^{\prime}}$ can be evaluated as follows. Since $\Gamma_{h}$ is piecewise planar and $\mathbf{v} \in \mathbf{V}_{i}$ is a piecewise quadratic function, for $\mathbf{v} \in \mathbf{V}_{i}$, both $\hat{f}_{\Gamma_{h}}(\mathbf{v})$ and $f_{\Gamma_{h}}(\mathbf{v})$ can be computed exactly (up to machine accuracy) using suitable quadrature rules. For the evaluation of the dual norm $\|\cdot\|_{\mathbf{V}_{i}^{\prime}}$ we proceed as follows. Let $\left\{\boldsymbol{\phi}_{j}\right\}_{j=1, \ldots, n}$ be the 
standard nodal basis in $\mathbf{V}_{i}$ and $J: \mathbb{R}^{n} \rightarrow \mathbf{V}_{i}$ the isomorphism $J \vec{x}=\sum_{k=1}^{n} x_{k} \boldsymbol{\phi}_{k}$. Let $M_{h}$ be the mass matrix and $A_{h}$ the stiffness matrix of the Laplacian:

$$
\begin{gathered}
\left(M_{h}\right)_{i, j}:=\int_{\Omega} \phi_{i} \cdot \phi_{j} d x, \quad 1 \leq i, j \leq n . \\
\left(A_{h}\right)_{i, j}:=\int_{\Omega} \nabla \phi_{i} \cdot \nabla \phi_{j} d x .
\end{gathered}
$$

Define $C_{h}=A_{h}+M_{h}$. Note that for $\mathbf{v}=J \vec{x} \in \mathbf{V}_{i}$ we have $\|\mathbf{v}\|_{1}^{2}=\left\langle C_{h} \vec{x}, \vec{x}\right\rangle$. Take $e \in \mathbf{V}_{i}^{\prime}$ and define $\vec{e} \in \mathbb{R}^{n}$ by $e_{j}:=e\left(\phi_{j}\right), j=1, \ldots, n$. Due to

$$
\|e\|_{\mathbf{V}_{i}^{\prime}}=\sup _{\mathbf{v} \in \mathbf{V}_{i}} \frac{|e(\mathbf{v})|}{\|\mathbf{v}\|_{1}}=\sup _{\vec{x} \in \mathbb{R}^{n}} \frac{\left|\sum_{j=1}^{n} x_{j} e\left(\boldsymbol{\phi}_{j}\right)\right|}{\sqrt{\left\langle C_{h} \vec{x}, \vec{x}\right\rangle}}
$$

we obtain

$$
\|e\|_{\mathbf{V}_{i}^{\prime}}=\sup _{\vec{x} \in \mathbb{R}^{n}} \frac{\langle\vec{x}, \vec{e}\rangle}{\sqrt{\left\langle C_{h} \vec{x}, \vec{x}\right\rangle}}=\left\|C_{h}^{-1 / 2} \vec{e}\right\|=\sqrt{\left\langle C_{h}^{-1} \vec{e}, \vec{e}\right\rangle}
$$

Thus for the computation of $\|e\|_{\mathbf{V}_{i}^{\prime}}$ we proceed in the following way:

1. Compute $\vec{e}=\left(e\left(\phi_{j}\right)\right)_{j=1}^{n}$.

2. Solve the linear system $C_{h} \vec{z}=\vec{e}$ up to machine accuracy.

3. Compute $\|e\|_{\mathbf{V}_{i}^{\prime}}=\sqrt{\langle\vec{z}, \vec{e}\rangle}$.

We applied this strategy to $e:=\hat{f}_{\Gamma_{h}}-f_{\Gamma_{h}}$. The results are given in the second column in table 6.1. The numerical order of convergence in the third column of this table clearly indicates an $\mathcal{O}(\sqrt{h})$ behaviour. Due to (6.7) this implies the same $\mathcal{O}(\sqrt{h})$ convergence behaviour for $\left\|f_{\Gamma}-f_{\Gamma_{h}}\right\|_{\mathbf{V}_{i}^{\prime}}$. This indicates that the $\mathcal{O}(\sqrt{h})$ bound in Corollary 4.8 is sharp.

The same procedure can be applied with $f_{\Gamma_{h}}$ replaced by the modified (improved) approximate surface tension force

$$
\tilde{f}_{\Gamma_{h}}(\mathbf{v})=\tau \sum_{i=1}^{3} \tilde{g}_{h, i}\left(v_{i}\right)
$$

with $\tilde{g}_{h, i}$ as defined in (5.2). This yields the results in the fourth column in table 6.1. For this modification the numerical order of convergence is significantly better, namely at least first order in $h$. From (6.7) it follows that for $\left\|f_{\Gamma}-\tilde{f}_{\Gamma_{h}}\right\|_{\mathbf{V}_{i}^{\prime}}$ we can expect $\mathcal{O}\left(h^{p}\right)$ with $p \geq 1$.

Summarizing, we conclude that the results of these numerical experiments confirm the theoretical $\mathcal{O}(\sqrt{h})$ error bound derived in the analysis in section 4 and show that the modified approximation indeed leads to (much) better results.

\section{REFERENCES}

[1] E. BÄNSCH, Numerical methods for the instationary Navier-Stokes equations with a free capillary surface, habilitation thesis, University of Freiburg, 1998.

[2] - Finite element discretization of the Navier-Stokes equations with a free capillary surface, Numer. Math., 88 (2001), pp. 203-235.

[3] J. U. Brackbill, D. B. Kothe, and C. ZEmach, A continuum method for modeling surface tension, J. Comp. Phys., 100 (1992), pp. 335-354. 


\begin{tabular}{|c||c|c||c|c|}
\hline$i$ & $\left\|\hat{f}_{\Gamma_{h}}-f_{\Gamma_{h}}\right\|_{\mathbf{V}_{i}^{\prime}}$ & order & $\left\|\hat{f}_{\Gamma_{h}}-\tilde{f}_{\Gamma_{h}}\right\|_{\mathbf{V}_{i}^{\prime}}$ & order \\
\hline 0 & $1.79 \mathrm{E}-1$ & - & $1.32 \mathrm{E}-1$ & - \\
1 & $1.40 \mathrm{E}-1$ & 0.35 & $4.43 \mathrm{E}-2$ & 1.57 \\
2 & $1.03 \mathrm{E}-1$ & 0.45 & $1.46 \mathrm{E}-2$ & 1.61 \\
3 & $7.22 \mathrm{E}-2$ & 0.51 & $5.06 \mathrm{E}-3$ & 1.52 \\
4 & $5.02 \mathrm{E}-2$ & 0.53 & $1.78 \mathrm{E}-3$ & 1.51 \\
\hline
\end{tabular}

Error norms and numerical order of convergence for different refinement levels.

[4] Y. C. Chang, T. Y. Hou, B. Merriman, and S. Osher, A level set formulation of Eulerian interface capturing methods for incompressible fluid flows, J. Comp. Phys., 124 (1996), pp. 449-464.

[5] K. Deckelnick and G. Dziuk, Mean curvature flow and related topics, in Frontiers in Numerical Analysis, Durham 2002, J. F. Blowey, A. W. Craig, and T. Shardlow, eds., Springer, 2003, pp. 63-108.

[6] A. Demlow and G. Dziuk, An adaptive finite element method for the Laplace-Beltrami operator on implicitly defined surfaces, Preprint 06-02, IAM, University of Freiburg, 2006. Accepted for publication in SIAM J. Numer. Anal.

[7] DROPS package. http://www.igpm.rwth-aachen.de/DROPS/.

[8] G. DzIUK, An algorithm for evolutionary surfaces, Numer. Math., 58 (1991), pp. 603-611.

[9] S. Ganesan, G. Matthies, and L. Tobiska, On spurious velocities in incompressible flow problems with interfaces, Preprint 05-35, Department of Mathematics, University of Magdeburg, 2005.

[10] S. GANESAN AND L. Tobiska, Finite element simulation of a droplet impinging a horizontal surface, in Proceedings of ALGORITMY 2005, 2005, pp. 1-11.

[11] V. Girault and P. A. Raviart, Finite Element Methods for Navier-Stokes Equations, Springer, Berlin, 1986.

[12] S. Gross, V. Reichelt, And A. Reusken, A finite element based level set method for two-phase incompressible flows, Preprint 243, IGPM, RWTH Aachen, 2004. Accepted for publication in Comp. Visual. Sci. 2006.

[13] S. Gross And A. Reusken, Parallel multilevel tetrahedral grid refinement, SIAM J. Sci. Comput., 26 (2005), pp. 1261-1288.

[14] S. Hysing, A new implicit surface tension implementation for interfacial flows, Preprint 295, Department of Mathematics, University of Dortmund, 2005. Submitted to Int. J. Numer. Meth. Fluids.

[15] M. A. Olshanskit, J. Peters, and A. Reusken, Uniform preconditioners for a parameter dependent saddle point problem with application to generalized Stokes interface equations, Preprint 252, IGPM, RWTH Aachen, 2005. Accepted for publication in Numer. Math.

[16] M. A. Olshanskit and A. Reusken, A Stokes interface problem: stability, finite element analysis and a robust solver, in Proc. of European Congress on Computational Methods in Applied Sciences and Engineering, ECCOMAS 2004, P. Neittaanmäki et al., eds., 2004.

[17] - Analysis of a Stokes interface problem, Numer. Math., 103 (2006), pp. 129-149.

[18] S. Osher AND R. P. Fedkiw, Level set methods: An overview and some recent results, J. Comp. Phys., 169 (2001), pp. 463-502.

[19] S. P. van der Pijl, A. Segal, C. Vuik, and P. Wesseling, A mass-conserving level-set method for modelling of multi-phase flows, Int. J. Num. Meth. Fluids, 47 (2005), pp. 339361.

[20] S. B. Pillapakkam And P. Singh, A level-set method for computing solutions to viscoelastic two-phase flow, J. Comp. Phys., 174 (2001), pp. 552-578.

[21] J. A. Sethian, Level set methods and fast marching methods, Cambridge University Press, 1999.

[22] M. Sussman, A. S. Almgren, J. B. Bell, Ph. Colella, L. H. Howell, and M. L. Welcome, An adaptive level set approach for incompressible two-phase flows, J. Comp. Phys., 148 (1999), pp. 81-124.

[23] M. Sussman, P. Smereka, And S. Osher, A level set approach for computing solutions to incompressible two-phase flow, J. Comp. Phys., 114 (1994), pp. 146-159.

[24] A.-K. TornberG, Interface tracking methods with application to multiphase flows, doctoral 
thesis, Royal Institute of Technology, Department of Numerical Analysis and Computing Science, Stockholm, 2000.

[25] A.-K. Tornberg And B. Engquist, A finite element based level-set method for multiphase flow applications, Comp. Vis. Sci., 3 (2000), pp. 93-101.

[26] X. Yang, A. J. James, J. Lowengrub, X. Zheng, and V. Cristini, An adaptive coupled level-set/volume-of-fluid interface capturing method for unstructured triangular grids, J. Comp. Phys., Article in press (2006).

[27] X. Zheng, A. Anderson, J. Lowengrub, and V. Cristini, Adaptive unstructured volume remeshing II: Application to two- and three-dimensional level-set simulations of multiphase flow, J. Comp. Phys., 208 (2005), pp. 191-220. 